D. Nutzinger (Vienna) reported effects and problems encountered in combined drug and psychotherapy treatment. Most studies are based on an additive model tested by $2 \times 2$ designs, but only very few include a third group i. e. a placebo pill and placebo psychotherapy group as additional controls. Besides several other studies, a combined fluphenazine and behavior therapy study confirmed the assumption that medical treatment is superior to psychotherapy, however the combination of both is more effective than single treatment procedures. The discussion emphasized that uncontrollable factors like self-selection of patients and previous exposure to psychotherapy are hard to control, but that in many patients additional medication is the only way to render extreme distress accessible to psychotherapy.

The many difficulties encountered in the evaluation of multicenter studies were demonstrated by $\mathrm{H}$. Katschnig (Vienna) who reffered to the results of a cross national study with 12 collaborating centres comparing imipramine, alprazolam, and placebo. Data revealed that differences between centres and countries mainly referred to severity and type of panic attacks. This can be partially explained by linguistic discrepancies and cross cultural differences in the symptomatology of panic and anxiety. Dropout rates were found to differ considerably between centres; both active compounds turned out to be superior compared to placebo except for the United Kingdom, where no significant differences between placebo and alprazolam were found. The discussion was concerned with problems of stratified or metaanalyses in multicenter studies and coping with heterogeneities derived from differences in non-drug therapeutic factors (e. g. endeavour to keep patients in the study protocol).

\section{Speech Chronemics in Drug Evaluation}

\section{H. P. Krüger}

Psychologisches Institut der Universität Würzburg

and E. M. Bonerad, H. J. Dennler, E. Dunkl, H. J. Friese, Ch. Hain, J. Kazenwadel, R. Kohnen, W. Maier, M. Menzel, G. E. Trott, M. Vollrath

Expressive behavior must be described in a threefold manner: first there is the ACTOGRAM of behavior which includes the obvious meaning of an expression (the semantics). Second, all expressive behavior has topological consequences, i. e. variations in space - the TOPOGRAM. Finally, behavior has a temporal structure - the CHRONOGRAM. This last describes the temporal features of behavior as well as clarifying the communicative meaning which the temporal structure of an expressive act has.

Speaking as an expressive act can only occur sequentially, (whereas spatial relations exist simultaneously). Sounds must be produced one at a time, hence the communication between two persons must be organized in a 'now me - then you' sequence. Therefore, the psychological processes which structure time can best be analyzed in speech. This is done by SPEECH CHRONEMICS, a discipline which from a methodological viewpoint has to point out that time viewed psychologically is quite different from the time concept in physics. Psychological time as it is realized in the temporal structure of speaking must be understood as an ON-OFF pattern which is hierarchically ordered into four time levels:

- the MACRO level which discriminates the ON-OFF of daily periods when conversations occur and when they do not. "Zooming" inside one conversation yields a new ONOFF, the

- the UPPER MESO LEVEL. ON-OFFs within one conversation are utterances and the respective pauses. In a still more detailed look (in chronography this is done by a faster resolution of the signal) one utterance is

- at the LOWER MESO LEVEL, a new ON-OFF ensemble of vocalizations and pauses between them (duration: shorter than 1 second). Finally, a single vocalization is at the

- MICRO level an ON-OFF pattern of phonations and articulation pauses (shorter than 200 milliseconds).

The workshop dealt with the question of whether these different time levels have special validities in psychopharmacological research. All contributions used the same methodology: the analysis of the time structure of speech was done by the LOGOPORT, a device described in Krüger (Pharmacopsychiatry, 22 (I), 1989 5-12).

Hain \& Maier (Mainz) assessed various speech parameters at the micro and meso level in a longitudinal investigation of 18 initially unmedicated patients with major depression and 8 healthy controls. On admission, prolonged pause times during effortful counting ("count as fast as you can") discriminated best between the two groups. Significant correlations were found between the pause times and scores on the HAMD and Widlocher scale, notably the motor-retardation items. During successful antidepressant pharmacotherapy, the pause times above a cutoff level of 200 ms (meso level) were shortened, whereas the pauses below this level (articulatory level) became slightly longer. In some cases the change of speech parameters came before the clinical improvement, indicating their predictive potential. Apart from their use for the evaluation of antidepressant therapy, speech recordings may be a valuable tool for the objective monitoring of the psychomotor state in other conditions such as negative schizophrenia.

These results at the articulatory level agreed with those of Vollrath (Würzburg). It has long been understood that the pronounciation of a stop consonant in the context vowel-consonant (as in/ak/) yields an articulation pause (AP) caused by a brief closure of the oral cavity. Within the context consonant-vowel (as in $/ \mathrm{ka} /$ ) the voiceonset time (VOT) is observed which corresponds to the time needed to switch frorii the consonant to the vowel. Psycholinguistic resaerch found only small variation between subjects in the duration of the AP and VOT when a certain stop consonant is produced in a stable prosodic environment. This stability is due to the fact that the duration of these parameters is an important cue for the perception and the distinction of different stop-consonants. Many speech disorders are 
characterized by altered durations of the AP and VOT. Despite this clinical evidence, no systematic research has been done concerning the question whether variations in the duration of the AP or VOT are associated with different states of arousal.

To investigate this hypothesis, Vollrath observed the production of an AP and VOT under highly controlled conditions. In a placebo-controlled study the duration of the AP decreased significantly under the influence of caffeine. However, there was no significant change in the VOT. A case study investigated these parameters under three different levels of blood alcohol concentration (BAC). The duration of the AP increased during the rise-time of the BAC and decreased when the peak of the BAC was passed. Without alcohol, there was no comparable trend. Again, there was no systematic effect on the VOT. These results are encouraging for the use of the AP as a new and sensitive methodological tool. However, further research has to be done to clarify the underlying psychophysiological processes.

The use of chronemic parameters in the study of hyperactive children was demonstrated by Trott, Menzel, Bonerad \& Friese (Würzburg). In judging the course of treatment of hyperkinetic syndrome, psychiatrists have hitherto preferred rating scales to get a global activity evaluation at the behavioral level. In addition, objective psychometric tests were given under controlled conditions. This last have proven useless in the laboratory, as hyperkinetic children behave relatively normally in low-stimulus environments. The authors have used chronemic parameters to study the therapeutic effectiveness of Moclobemid among 20 hyperkinetic children. Speech measurements were made with the LOGOPORT in situations of everyday life. The duration and the amount of speaking activity decreased, while the intervals between conversations grew longer. ACTOMETER readings on motor unrest showed a decrease in gross motor activity, especially in specifically disturbing situations such as school lessons, but also during sleep. For evaluating fine motor actions with this group of patients standardized analyses in the laboratory have proven useful.

A combination of measures for speaking and motor activity also was used by Kazenwadel, Dennler, Dunkl \& Kohnen (Würzburg/Nürnberg). In a placebo-controlled, double-blind and randomized experiment, the authors investigated the effects of Hydergin i. v. $(10 \mathrm{ml})$ on motor and social activity of 80 geriatric outpatients. Patients received infusion treatments 2 hours a day for 3 weeks. Synchronous measurements of speaking and motor activity were recorded in situations of everyday life with portable devices (LOGOPORT and ACTIGRAPH) on days 1, 11, and 22 of treatment. A preliminary statistical analysis of the final measurements on day 22 revealed no differences in motor activity, but higher social activity (i. e., more and longer conversations) in Hydergin-treated patients, compared to the placebo group.

Bivariate analysis of both parameters indicated that the observed changes in speaking activity are not caused by a general increase of motor activity. Under verum treatment, motor activity is reduced, if at all, only in "non-social" situations. Thus, the behavioral effects of Hydergin i. v. are more adequately interpreted in terms of emotional and/or cognitive effects.

Conclusions: The contributions proved that the chronemic structure of behavior - especially the time pattern of speech - is a promising tool in the evaluation of drug effects. It was shown that, whereas micro processes mostly refer to psychomotoric changes, the meso and macro level may be used as indicators for cognitive and emotional effects. The parallel use of motor and verbal behavior-monitoring devices seems to be especially fruitful for the understanding of drug effects. 